\title{
Condições clínicas e perfil metabólico de prematuros do nascimento aos seis meses de idade corrigida
}

\author{
Angela Israel Graeff Borges ${ }^{1}$, \\ Sandra Lucinei Balbo², \\ Maristela Salete Maraschin ${ }^{3}$, \\ Grasiely Masotti Scalabrin Barreto ${ }^{4}$, \\ Beatriz Rosana Gonçalves de Oliveira Toso ${ }^{5}$, \\ Cláudia Silveira Viera ${ }^{6}$
}

\section{RESUMO}

A sobrevida de recém-nascidos prematuros e de baixo peso tem evidenciado alterações metabólicas ao longo de suas vidas. Objetiva-se correlacionar se condições clínicas do recém-nascido prematuro (RNPT) ao nascimento, na hospitalização e após a alta da Unidade de Terapia Intensiva Neonatal, influenciam no seu perfil metabólico aos seis meses de Idade Corrigida. Estudo prospectivo, de coorte, com 37 RNPT. A análise estatística realizada foi descritiva e inferencial. Ao correlacionar as condições clínicas do nascimento, da hospitalização e do seguimento com o perfil metabólico do RNPT, evidenciou-se que a enterocolite necrotizante $(p=0,006)$ e sepse tardia $(p=0,02)$ apresentaram diferença estatística significativa na concentração de insulina. O perfil glicêmico na presença das comorbidades se manteve normal e o perfil lipídico elevou-se gradativamente. Os RNPT deste estudo constituemse em grupo de risco para o desenvolvimento da síndrome metabólica e doenças cardiovasculares, devido às alterações lipídicas e insulínicas encontradas.

Descritores: Recém-Nascido Prematuro; Enfermagem Pediátrica; Unidades de Terapia Intensiva Neonatal; Continuidade da Assistência ao Paciente; Síndrome Metabólica.

\footnotetext{
${ }^{1}$ Enfermeira. Enfermeira Responsável Técnica da Universidade Paranaense. Cascavel, PR, Brasil. E-mail: angelagraeff15@gmail.com.

2 Bióloga, Doutora em Ciências Biológicas. Professora Associada da Universidade Estadual do Oeste do Paraná, Campus Cascavel. Cascavel, PR, Brasil. E-mail: slbalbo@hotmail.com.

${ }^{3}$ Enfermeira, Mestre em Enfermagem. Docente da Universidade Estadual do Oeste do Paraná, Campus Cascavel. Cascavel, PR, Brasil. E-mail: maraschinmaristela@gmail.com.

${ }^{4}$ Enfermeira, Mestre em Biociências e Saúde. Enfermeira do Hospital Universitário do Oeste do Paraná, Campus Cascavel. Cascavel, PR, Brasil. Email: grasi-barreto@hotmail.com.

${ }^{5}$ Enfermeira, Doutora em Enfermagem em Saúde Pública. Professora Adjunta da Universidade Estadual do Oeste do Paraná, Campus Cascavel. Cascavel, PR, Brasil. E-mail: grasi-barreto@hotmail.com.

${ }^{6}$ Enfermeira, Doutora em Enfermagem em Saúde Pública. Professora Adjunta da Universidade Estadual do Oeste do Paraná, Campus Cascavel. Cascavel, PR, Brasil. E-mail: clausviera@gmail.com.
}

Artigo recebido: 14/09/2017. Artigo aprovado: 23/05/2018. Artigo publicado: 05/12/2018.

\section{Como citar esse artigo:}

Borges AIG, Balbo SL, Maraschin MS, Barreto GMS, Toso BRGO, Viera CS. Condições clínicas e perfil metabólico de prematuros do nascimento aos seis meses de idade corrigida. Rev. Eletr. Enf. [Internet]. 2018 [acesso em:

];20:v20a30. Disponível em: https://doi.org/10.5216/ree.v20.49352. 


\section{INTRODUÇÃO}

Mundialmente, 15 milhões de bebês nascem prematuramente ao ano, o Brasil aparece em 10 lugar, com 279.000 partos prematuros anualmente. No período de 2011 a 2012, a taxa brasileira de nascimentos prematuros foi de $11,5 \%$, com variação de $10,4 \%$ a $13 \%$ entre as distintas regiões do país. Na região Sul, onde se localiza o município em que o estudo foi desenvolvido, os nascimentos prematuros compreendem $11,2 \%$ do total de nascimentos ${ }^{(1)}$. Em decorrência desse percentual de nascimentos prematuros, tem-se o incremento da sobrevivência de Recém-nascidos Prematuros (RNPT) cada vez de menor Idade Gestacional (IG) e menor peso ao nascer.

Ademais, a prematuridade traz consequências às famílias e à sociedade em geral, decorrente do custo social e financeiro de difícil mensuração, bem como, tem repercussões para a saúde da criança desde a hospitalização na Unidade de Terapia Intensiva Neonatal (UTIN) como ao longo de sua vida após a alta hospitalar.

O RNPT sofre influência dos fatores nutricionais e metabólicos maternos intraútero, bem como das condições clínicas do período gestacional e, ao nascer passa a sofrer ações do ambiente extrauterino em seu comportamento fisiológico e emocional. Durante o período pós-natal, esse bebê está sujeito a inúmeras complicações, dentre as quais vem sendo estudada, nas últimas décadas, a associação entre prematuridade e o desenvolvimento de agravos como hipertensão arterial, intolerância à glicose e dislipidemias, tanto na infância, adolescência como também na idade adulta ${ }^{(2)}$.

As evidências apontam que a relação do nascimento prematuro e o ganho de peso da criança nas primeiras semanas está diretamente associado ao aumento do sobrepeso e obesidade infantil a partir dos cinco anos de idade, bem como a alterações da pressão arterial, insulina plasmática e na leptina de adolescentes ${ }^{(3-4)}$. No entanto, estudos que correlacionem as condições clínicas e o perfil metabólico do RNPT ao nascimento e no seguimento após a alta hospitalar no Brasil, ainda são uma lacuna na literatura científica.

Considerando que o Brasil passa por um período de transição epidemiológica, em que há o crescimento dos casos de doenças crônicas não transmissíveis, as quais demandam longos tratamentos ${ }^{(5)}$ e, consequentemente, com aumento do adoecimento e a mortalidade da população, torna-se necessário a realização de pesquisas que identifiquem nas populações de risco, como o prematuro e baixo peso ao nascer, fatores que possam ser marcadores para as doenças crônicas não transmissíveis. Contribuindo assim para a redução do número de adultos com condições crônicas de saúde que impactam tanto na saúde da família, do indivíduo, como no sistema de saúde. O enfermeiro, como membro da equipe multiprofissional, tanto dos ambulatórios de seguimento como da atenção primária à saúde, pode contribuir para essa avaliação durante as consultas de enfermagem dos RNPT no seguimento após a alta hospitalar.

Questiona-se, então, se a condição metabólica aos seis meses de Idade Corrigida (IC) pode ser influenciada pelas condições clínicas do RNPT ao nascimento, na hospitalização e após a alta da UTIN. Para tanto, objetiva-se estabelecer a relação das condições clínicas do RNPT ao nascimento, na hospitalização e após a alta da UTIN com seu perfil metabólico aos seis meses de idade corrigida.

\section{METODOLOGIA}

Estudo de coorte, prospectivo, integrante do projeto de pesquisa "Repercussões da prematuridade: estresse materno e programação metabólica", aprovado no Edital Universal 014/2014, CNPq. Foi realizado no 
período de junho de 2015 a agosto de 2016 na UTIN de um hospital universitário do Paraná e no ambulatório de seguimento do referido hospital.

O presente estudo foi avaliado pelo Comitê de Ética da Universidade Estadual do Oeste do Paraná (CEP UNIOESTE), com aprovação sob o parecer no 385.370 obedecendo a resolução 466/2012 que regulamenta a Pesquisa com Seres Humanos.

A população constituiu-se de todos os RNPT que estiveram hospitalizados na UTIN de junho de 2015 até março de 2016 e retornaram no seguimento ao ambulatório de alto risco até agosto de 2016, contemplados nos critérios de inclusão: prematuros com até 36 semanas e seis dias de idade gestacional; sem anomalias congênitas. Excluídos aqueles que foram a óbito durante o período de hospitalização ou seguimento ou não compareceram na consulta de retorno no ambulatório. Assim, a amostra do estudo constituiu-se de 37 RNPT.

A captação dos participantes era feita junto à puérpera na maternidade, a qual era contatada entre 24 e 48 horas após o parto por um dos pesquisadores, explicando-lhe sobre a pesquisa. Com o aceite de participação e assinatura do Termo de Consentimento Livre e Esclarecido, iniciava-se a coleta de dados documentais e amostras do material bioquímico. Este material era obtido das sobras das amostras de sangue das coletas de rotina da unidade de centro obstétrico (mãe) e da UTIN (RNPT). O retorno dos RNPT no ambulatório era agendado pela enfermeira da UTIN, na alta da unidade. A consulta era realizada pela equipe da pesquisa aos seis meses de IC, contemplando a entrevista da mãe e avaliação clínica do RNPT, com coleta de amostras de material para realização dos exames bioquímicos desse período.

Os exames bioquímicos obtidos no nascimento, na alta da UTIN e no seguimento no ambulatório, foram insulina, glicose, colesterol total e triglicerídeos plasmáticos. A leitura dos exames foi realizada pelo método da química seca, na medição quantitativa da sua concentração no soro utilizando os Sistemas Químicos VITROS 250/350/950/5,1 FS e 4600 e o sistema Integrado VITROS 5600 (s/d). As dosagens de insulina foram realizadas pelo método de eletroquimioluminescência e apresentadas em unidades internacionais ( $\mathrm{uUI} / \mathrm{mL}$ ).

As variáveis analisadas referiram-se a três momentos:

- Nascimento: peso, Idade Gestacional (IG), gênero, relação de peso ao nascimento e IG, perfil lipídico, glicêmico e insulínico.

A classificação quanto ao grau de prematuridade refere-se: menor de 28 semanas de IG (prematuridade extrema); de 28 a 31 semanas (muito prematuro) e de 32 a 36 semanas e seis dias (prematuro moderado e tardio) ${ }^{(6)}$. Para classificar a adequação do peso ao nascimento foi utilizada a curva de Fenton e Kim ${ }^{(7)}$, específica para a população de prematuros, classificando-os em: Pequeno para a Idade Gestacional (PIG - aqueles abaixo do percentil 10), Grande para a Idade Gestacional (GIG - aqueles acima do percentil 90) e Adequado para Idade Gestacional (AIG- entre os percentis 10 e 90).

- Alta hospitalar: Perfil lipídico, glicêmico, insulínico e as comorbidades no período de hospitalização na UTIN.

- Seis meses de IC: Perfil lipídico, glicêmico e insulínico e comorbidades apresentadas no período pós-alta hospitalar, obtidas pelo diagnóstico médico.

As comorbidades analisadas referem-se a: síndrome do esforço respiratório, sepses precoce e tardia, Enterocolite Necrotizante (ECN), hemorragia periventricular, leucomalácia, ventriculomegalia, Displasia Broncopulmonar (DBP) e Retinopatia da Prematuridade (ROP). Os tratamentos a que o RNPT foi submetido na 
hospitalização referiram-se ao uso de oxigenioterapia, transfusão sanguínea e tratamento a laser da retinopatia da prematuridade.

Salienta-se que não há valores de referência específicos para o RNPT para os exames bioquímicos analisados neste estudo. Deste modo, os resultados dos parâmetros plasmáticos de glicose, colesterol e triglicerídeos foram comparados aos dados de referência da Sociedade Brasileira de Diabetes ${ }^{(8)}$ e ao Consenso Brasileiro para a normatização da determinação laboratorial do perfil lipídico ${ }^{(9)}$. Os valores de insulina embasaramse na referência do laboratório em que o exame foi processado.

Os dados foram digitados no programa Microsoft Excel Windows 2010 com dupla conferência. A análise estatística descritiva foi utilizada para caracterizar a amostra e o perfil metabólico, por meio de frequência absoluta e relativa, média e desvio padrão. A estatística inferencial foi aplicada para fazer a correlação entre as variáveis clínicas dos RNPT e seu perfil metabólico em que o padrão de distribuição dos resíduos foi realizado pelo teste de Shapiro-Wilk, bem como avaliada a homogeneidade das variâncias pelo teste de Cochran. Uma vez que os dados se encontravam em acordo com tais pressupostos, foi aplicado o teste ANOVA fatorial para medidas repetidas, seguido pelo teste de acompanhamento de LSD-Fisher. Todas as análises foram realizadas no programa estatístico STATISTICA $7.0^{\circledR}$.

Quanto às comorbidades: síndrome do desconforto respiratório, leucomalácia severa, ventriculomegalia, as análises não puderam ser realizadas devido ao número insuficiente de pacientes com ou sem as patologias ou uso de tratamento.

\section{RESULTADOS}

No período do estudo, nasceram 47 RNPT, destes, oito foram a óbito no período neonatal precoce e dois no período neonatal tardio. Sendo, portanto, excluídos do presente estudo, constituindo-se a amostra final de 37 RNPT acompanhados desde o nascimento até os seis meses de IC no ambulatório de seguimento. Apresenta-se a caracterização da amostra em relação às variáveis do nascimento, as quais correspondem ao gênero, peso, relação peso de nascimento/IG e IG (Tabela 1 ).

Tabela 1: Características dos RNPT acompanhados quanto aos dados de nascimento (n=37). Cascavel, PR, Brasil. 2016.

\begin{tabular}{|c|c|c|}
\hline Variáveis & FA & FR (\%) \\
\hline \multicolumn{3}{|l|}{ Gênero } \\
\hline Masculino & 18 & $48,60 \%$ \\
\hline Feminino & 19 & $51,30 \%$ \\
\hline \multicolumn{3}{|l|}{ Peso } \\
\hline$\leq 1.000 \mathrm{~g}$ & 4 & $10,80 \%$ \\
\hline 1.000 a $1.499 \mathrm{~g}$ & 15 & $40,50 \%$ \\
\hline $1.500 \mathrm{a} \geq 2.500 \mathrm{~g}$ & 18 & $48,60 \%$ \\
\hline \multicolumn{3}{|l|}{ Relação Peso/IG } \\
\hline Pequeno à Idade Gestacional & 4 & $10,80 \%$ \\
\hline Adequado à Idade Gestacional & 32 & $86,50 \%$ \\
\hline Grande à Idade Gestacional & 1 & $2,70 \%$ \\
\hline \multicolumn{3}{|l|}{ IG } \\
\hline$<28$ sem & 4 & $10,80 \%$ \\
\hline 28-31 sem & 15 & $40,50 \%$ \\
\hline $32-36$ sem & 18 & $48,60 \%$ \\
\hline
\end{tabular}

Legenda: FA: Frequência absoluta; FR: Frequência Relativa; IG: Idade Gestacional 
Os RNPT estudados se caracterizaram por não haver predominância quanto ao sexo, sendo balanceado entre feminino $(n=19 / 51,3 \%)$ e masculino $(n=18 / 48,6 \%)$, em sua maioria com peso ao nascer inferior a 1.500 gramas ( $n=19 / 51,3 \%)$ e em sua maioria $(n=32 / 86,5 \%)$ classificados quanto à adequação de peso ao nascer como AIG, ou seja, encontravam-se acima do percentil 10 da curva de referência. Quanto a IG, 18 (48,6\%) eram muito prematuros, sendo a média da IG ao nascimento de $31( \pm 5,8)$ semanas. O tempo de hospitalização está de acordo com a classificação do grau de prematuridade encontrada, visto que a média de tempo de hospitalização de 21 $( \pm 14,6)$ dias condiz com RNPT muito prematuros e moderadamente prematuros (Tabela 1 ).

Na Tabela 2 apresentam-se as principais comorbidades identificadas e tratamentos os quais os RNPT foram submetidos durante a internação.

Tabela 2: Descrição de comorbidades e tratamentos durante a internação de RNPT na UTIN (n=37). Cascavel, PR, Brasil. 2016.

\begin{tabular}{|c|c|c|}
\hline Variáveis & FA & $\mathrm{FR}(\%)$ \\
\hline \multicolumn{3}{|l|}{ Enterocolite necrotizante } \\
\hline Sim & 8 & $21,60 \%$ \\
\hline Não & 29 & $78,40 \%$ \\
\hline \multicolumn{3}{|l|}{ Síndrome do desconforto respiratório } \\
\hline Sim & 36 & $97,30 \%$ \\
\hline Não & 1 & $2,70 \%$ \\
\hline \multicolumn{3}{|l|}{ Hemorragia Perintraventricular } \\
\hline Sim & 2 & $5,40 \%$ \\
\hline Não & 35 & $94,60 \%$ \\
\hline \multicolumn{3}{|l|}{ Sepse precoce } \\
\hline Sim & 27 & $72,90 \%$ \\
\hline Não & 10 & $27,00 \%$ \\
\hline \multicolumn{3}{|l|}{ Sepse Tardia } \\
\hline Sim & 16 & $43,20 \%$ \\
\hline Não & 21 & $56,70 \%$ \\
\hline \multicolumn{3}{|l|}{ Retinopatia da prematuridade (ROP) } \\
\hline Sim & 6 & $16,20 \%$ \\
\hline Não & 31 & $83,80 \%$ \\
\hline \multicolumn{3}{|l|}{ Tratamento Laser ROP } \\
\hline Sim & 1 & $2,70 \%$ \\
\hline Não & 36 & $97,30 \%$ \\
\hline \multicolumn{3}{|l|}{ Displasia Broncopulmonar } \\
\hline Sim & 6 & $16,20 \%$ \\
\hline Não & 31 & $83,80 \%$ \\
\hline \multicolumn{3}{|l|}{ Dependência $\mathrm{O}_{2}$ na Alta } \\
\hline Sim & 11 & $29,70 \%$ \\
\hline Não & 26 & $70,30 \%$ \\
\hline
\end{tabular}

Legenda: FA: Frequência Absoluta; FR: Frequência Relativa; IG: Idade Gestacional

As principais comorbidades apresentadas no período da internação foram a síndrome do desconforto respiratório $(n=36 / 97,3 \%)$, sepse precoce $(n=27 / 72,9 \%)$, sepse tardia $(n=16 / 43,2 \%)$. Dentre os tratamentos a que o RNPT foi submetido, prevaleceu a manutenção da oxigenoterapia na alta da UTIN ( $n=11 / 29,7 \%$ ) (Tabela 2).

Na Tabela 3 apresenta-se o perfil metabólico por meio dos parâmetros plasmáticos de colesterol total, triglicerídeos, insulina e glicose do nascimento aos seis meses de IC dos RNPT.

O perfil metabólico dos RNPT estudados evidência que a glicemia mostrou estabilidade da admissão na UTIN até os seis meses de IC sem diferença estatisticamente significativa $(p=0,639)$. Contudo, conforme visto na 
Tabela 3 o perfil lipídico $(p=<0,0001)$ e insulínico $(p=0,048)$ apresentaram diferença estatística significativa para os períodos avaliados. A insulina apresentou redução do nascimento até o sexto mês de IC. No perfil lipídico, tanto o colesterol total como o triglicerídeo demonstraram elevação gradativa da admissão aos seis meses de IC.

Tabela 3: Parâmetros plasmáticos do perfil lipídico, glicêmico e insulínico dos RNPT do nascimento, na alta e aos seis meses de Idade Corrigida ( $n=37)$. Cascavel, PR, Brasil. 2016.

\begin{tabular}{|c|c|c|c|}
\hline Variáveis & Média (DP) & Min-Max & p-valor \\
\hline \multicolumn{4}{|l|}{ Glicemia (mg/dL) } \\
\hline Admissão & $83,11( \pm 8,20)$ & $20-285$ & \\
\hline Alta & $80,25( \pm 4,62)$ & $20-164$ & 0,639 \\
\hline 6 meses & $87,53( \pm 2,41)$ & $64-132$ & \\
\hline \multicolumn{4}{|l|}{ Triglicerídeos $(\mathrm{mg} / \mathrm{dL})$} \\
\hline Admissão & $45,36( \pm 4,72)^{a}$ & $16-139$ & \\
\hline Alta & $87,17(+6,0)^{b}$ & $34-178$ & $<0,0001$ \\
\hline 6 meses & $153,7(+13,2)^{c}$ & $46-338$ & \\
\hline \multicolumn{4}{|l|}{ Colesterol Total (mg/dL) } \\
\hline Admissão & $77,97( \pm 4,13)^{c}$ & $25-130$ & \\
\hline Alta & $114,83( \pm 5,8)^{b}$ & $64-215$ & $<0,0001$ \\
\hline 6 meses & $136,94( \pm 5,7)^{\mathrm{a}}$ & $83-240$ & \\
\hline \multicolumn{4}{|l|}{ Insulina (ulU/mL) } \\
\hline Admissão & $11,1(+2,5)^{b}$ & $0,81-83$ & \\
\hline Alta & $7,0(+1,45)^{\mathrm{ab}}$ & $0,36-45$ & 0,048 \\
\hline 6 meses & $5,0( \pm 0,66)^{a}$ & $1,2-16$ & \\
\hline
\end{tabular}

Legenda:

- DP: Desvio Padrão

- * Quando as médias foram estatisticamente diferentes nos três períodos avaliados, analisadas pelo teste de ANOVA são representadas na tabela pela junção das letras ab;

- $\quad$ ** Quando não houve diferença estatística entre as médias verificadas pelo teste de variância (ANOVA) nos três períodos avaliados, considerando-se o DP, são representadas na tabela pelas letras a; b ou c, isoladamente.

- Valores de referência utilizados: Glicemia $80-126 \mathrm{mg} / \mathrm{dL}$ (pós-prandial); Triglicerídeos <85mg/dL (sem jejum); Colesterol Total <170mg/dL (sem jejum); Insulina 2,6 a 24,9 uUl/mL.

Ademais, na descrição da amostra estudada nos três períodos de avaliação, a maioria dos RNPT apresentou concentração de insulina de acordo com os valores de referência. Em relação ao perfil glicêmico encontrou-se que, apesar de 15 recém-nascidos (40,5\%) terem apresentado hipoglicemia na admissão na UTIN, ao longo do seguimento esses valores foram se elevando, sendo que na alta da UTIN 17 (46,0\%) e aos seis meses de IC a maioria dos prematuros $(28 / 76,0 \%)$ apresentou índice glicêmico normal. Em relação ao perfil lipídico, na admissão bem como na alta da UTIN, grande parte dos RNPT estavam com valores adequados de triglicerídeos e colesterol total. Aos seis meses de IC, 20 (54,0\%) dos RNPT apresentaram elevação dos níveis séricos de triglicerídeos, a maioria com níveis $\geq 130 \mathrm{mg} / \mathrm{dl}$, caracterizando-se como trigliceridemia. $\mathrm{O}$ colesterol, no entanto, permaneceu em níveis normais para a maioria do RNPT ( $n=23 / 62,0 \%)$.

A correlação das variáveis acerca das comorbidades apresentadas pelos RNPT e o seu perfil bioquímico ao longo do seguimento estão apresentadas na Tabela 4.

Apenas a insulina apresentou diferença estatística significativa na presença das morbidades $\operatorname{ECN}(p=0,006)$ e sepse tardia ( $p=0,02$ ), nos períodos avaliados (Tabela 4). Para os RNPT que desenvolveram ECN, a insulina apresentou a maior média no período de admissão na UTIN $(21,6 \pm 26,8)$. Da mesma forma, na correlação com a sepse tardia, os níveis de insulina sérica elevados no período de admissão $(17,6 \pm 19,7)$, decresceram a valores 
muito menores nos momentos seguintes. Em relação a presença das comorbidades ROP, DBP e sepse precoce os valores da insulina se mostram estáveis com declínio a partir do momento de admissão (Tabela 4).

Tabela 4: Estatística descritiva (média \pm desvio padrão) e correlação do perfil lipídico, glicêmico e insulínico relacionados às comorbidades ECN, ROP, sepse precoce e tardia, DBP nos momentos de admissão, alta e seis meses de RNPT ( $n=37)$.

Cascavel, PR, Brasil. 2016.

\begin{tabular}{|c|c|c|c|c|c|}
\hline & ECN & Glicose & Triglicerídeos & Colesterol Total & Insulina \\
\hline \multirow{2}{*}{ Admissão } & Sim & $103,4 \pm 85,5$ & $43,4 \pm 39,9^{a}$ & $76,8 \pm 26,2^{b}$ & $21,6 \pm 26,8^{b}$ \\
\hline & Não & $77,3 \pm 33,0$ & $46,0 \pm 25,0^{\mathrm{a}}$ & $78,3 \pm 24,8^{b}$ & $8,1 \pm 7,8^{a}$ \\
\hline \multirow{2}{*}{ Alta } & Sim & $79,1 \pm 25,9$ & $80,2 \pm 29,7^{\mathrm{ab}}$ & $106,1 \pm 27,0^{\mathrm{a}}$ & $4,4 \pm 3,5^{a}$ \\
\hline & Não & $80,6 \pm 28,6$ & $89,1 \pm 37,9^{b}$ & $117,3 \pm 37,2^{\mathrm{a}}$ & $7,8 \pm, 6^{a}$ \\
\hline \multirow{3}{*}{6 meses } & Sim & $94,5 \pm 9,8$ & $138,4 \pm 85,1^{\mathrm{c}}$ & $117,2 \pm 20,6^{\text {ac }}$ & $4,1 \pm 2,2^{\mathrm{a}}$ \\
\hline & Não & $85,5 \pm 12,3$ & $158,1 \pm 78,5^{c}$ & $142,6 \pm 35,6^{c}$ & $5,2 \pm 4,4^{a}$ \\
\hline & $\begin{array}{c}\text { Sepse } \\
\text { precoce }\end{array}$ & Glicose & Triglicerídeos & Colesterol Total & Insulina \\
\hline \multirow{2}{*}{ Admissão } & Sim & $79,6 \pm 39,1$ & $46,0 \pm 29,8^{a}$ & $72,3 \pm 19,4^{b}$ & $9,4 \pm 9,6^{\mathrm{ab}}$ \\
\hline & Não & $93,5 \pm 73,9$ & $43,5 \pm 24,7 \underline{a}$ & $94,9 \pm 32,1^{\mathrm{ab}}$ & $16,1 \pm 25,3^{b}$ \\
\hline \multirow{2}{*}{ Alta } & Sim & $83,5 \pm 24,6$ & $89,9 \pm 38,0^{a}$ & $113,7 \pm 37,5^{a}$ & $7,3 \pm 9,4^{\mathrm{a}}$ \\
\hline & Não & $70,3 \pm 35,3$ & $79,0 \pm 29,9^{\mathrm{ab}}$ & $118,1 \pm 28,5^{\mathrm{ac}}$ & $6,0 \pm 6,4^{a}$ \\
\hline \multirow{2}{*}{6 meses } & Sim & $86,1 \pm 12,0$ & $151,7 \pm 81,8^{c}$ & $133,8 \pm 36,2^{\mathrm{cd}}$ & $5,5 \pm 4,2^{\mathrm{a}}$ \\
\hline & Não & $91,9 \pm 20,5$ & $159,8 \pm 75,0^{c}$ & $146,4 \pm 27,2^{d}$ & $3,6 \pm 2,8^{a}$ \\
\hline & Sepse tardia & Glicose & Triglicerídeos & Colesterol Total & Insulina \\
\hline \multirow{2}{*}{ Admissão } & Sim & $88,7 \pm 64,2$ & $49,9 \pm 28,5^{\mathrm{ab}}$ & $77,9 \pm 23,0^{\mathrm{a}}$ & $17,6 \pm 19,7^{b}$ \\
\hline & Não & $78,6 \pm 4,0$ & $41,7 \pm 28,3^{a}$ & $78,0 \pm 26,7^{a}$ & $5,8 \pm 6,4^{a}$ \\
\hline \multirow{2}{*}{ Alta } & Sim & $81,0 \pm 33,6$ & $90,2 \pm 38,4^{c}$ & $106,3 \pm 37,8^{b}$ & $7,9 \pm 11,6^{a}$ \\
\hline & Não & $79,7 \pm 22,8$ & $84,7 \pm 34,9^{b c}$ & $121,6 \pm 32,2^{b c}$ & $6,3 \pm 5,6^{a}$ \\
\hline \multirow{3}{*}{6 meses } & Sim & $86,0 \pm 18,2$ & $170,0 \pm 0,5^{d}$ & $132,4 \pm 25,4^{c d}$ & $4,5 \pm 3,3^{a}$ \\
\hline & Não & $88,8 \pm 10,9$ & $140,6 \pm 68,4^{d}$ & $140,5 \pm 40,2^{d}$ & $5,3 \pm 4,5^{a}$ \\
\hline & DBP & Glicose & Triglicerídeos & Colesterol Total & Insulina \\
\hline \multirow{2}{*}{ Admissão } & Sim & $75,2 \pm 36,4$ & $72,3 \pm 30,4^{\text {ac }}$ & $66,0 \pm 12,1^{c}$ & $10,7 \pm 9,8^{a b}$ \\
\hline & Não & $84,7 \pm 51,7$ & $40,0 \pm 25,0^{c}$ & $80,4 \pm 26,0^{c}$ & $11,2 \pm 16,0^{b}$ \\
\hline \multirow{2}{*}{ Alta } & Sim & $91,8 \pm 43,2$ & $122,2 \pm 46,0^{\mathrm{ab}}$ & $113,5 \pm 55,1^{\mathrm{ab}}$ & $12,6 \pm 16,9^{\mathrm{ab}}$ \\
\hline & Não & $78,0 \pm 24,0$ & $80,2 \pm 30,0^{\mathrm{a}}$ & $115,1 \pm 31,0^{\mathrm{a}}$ & $5,9 \pm 5,9^{a b}$ \\
\hline \multirow{3}{*}{6 meses } & Sim & $77,2 \pm 10,0$ & $177,0 \pm 93,6^{b}$ & $142,2 \pm 28,1^{\mathrm{ab}}$ & $3,3 \pm 2,7^{a b}$ \\
\hline & Não & $89,6 \pm 14,4$ & $149,0 \pm 77,0^{b}$ & $136,0 \pm 35,7^{b}$ & $5,3 \pm 4,1^{a}$ \\
\hline & ROP & Glicose & Triglicerídeos & Colesterol Total & Insulina \\
\hline \multirow{2}{*}{ Admissão } & Sim & $80,2 \pm 49,9$ & $44,2 \pm 27,4^{a}$ & $80,6 \pm 26,2^{b}$ & $10,9 \pm 16,0^{b}$ \\
\hline & Não & $97,8 \pm 47,2$ & $51,2 \pm 34,8^{\mathrm{ab}}$ & $64,8 \pm 7,4^{b}$ & $12,0 \pm 9,0^{\mathrm{ab}}$ \\
\hline \multirow{2}{*}{ Alta } & Sim & $78,0 \pm 24,7$ & $82,1 \pm 29,6^{b c}$ & $114,4 \pm 31,4^{c}$ & $6,2 \pm 6,0^{\mathrm{ab}}$ \\
\hline & Não & $91,3 \pm 40,7$ & $112,5 \pm 55,7^{c d}$ & $117,2 \pm 54,0^{\text {ac }}$ & $11,3 \pm 17,1^{\text {ab }}$ \\
\hline \multirow{2}{*}{6 meses } & Sim & $88,6 \pm 15,0$ & $146,4 \pm 74,3^{\text {de }}$ & $135,6 \pm 35,2^{\mathrm{a}}$ & $4,9 \pm 4,0^{\mathrm{a}}$ \\
\hline & Não & $82,3 \pm 11,2$ & $190,2 \pm 99,8^{e}$ & $143,7 \pm 31,2^{\mathrm{a}}$ & $5,5 \pm 4,5^{a b}$ \\
\hline
\end{tabular}

- $\quad$ ECN - Enterocolite necrotizante;

- ROP - Retinopatia da prematuridade;

- DBP - Displasia broncopulmonar.

- * Quando as médias foram estatisticamente diferentes nos três períodos avaliados, obtidas pelo teste de ANOVA são representadas na tabela pelas junção das letras ab; ac; bc; cd; de;

- $\quad$ ** Quando não houve diferença estatística significativa entre as médias nos três períodos avaliados, verificadas pelo teste de variância (ANOVA) são representadas na tabela pelas letras a; b; c; d; e, isoladamente.

- Valores de referência utilizados: Glicemia $80-126 \mathrm{mg} / \mathrm{dL}$ (pós-prandial); Triglicerídeos <85mg/dL (sem jejum); Colesterol Total <170mg/dL (sem jejum); Insulina 2,6 a 24,9 uUl/mL.

Os tratamentos a que foram submetidos os RNPT e o perfil bioquímico são descritos na Tabela 5. 
Tabela 5: Estatística descritiva (média \pm desvio padrão) das variáveis relacionadas a oxigênio na alta e transfusão sanguínea nos momentos de admissão, alta e seis meses dos RNPT relacionadas aos perfis glicêmicos, lipídico e insulínico ( $n=37$ ). Cascavel, PR, Brasil. 2016.

\begin{tabular}{|c|c|c|c|c|c|}
\hline & $\mathrm{O}^{2}$ alta & Glicose & Triglicerídeos & Colesterol Total & Insulina \\
\hline \multirow{2}{*}{ Admissão } & Sim & $87,8 \pm 42,0$ & $57,6 \pm 29,3^{a b}$ & $73,1 \pm 14,7^{b}$ & $14,2 \pm 11,7^{b}$ \\
\hline & Não & $81,0 \pm 52,7$ & $40,0 \pm 26,7^{a}$ & $80,1 \pm 28,1^{b}$ & $9,7 \pm 16,2^{\mathrm{ab}}$ \\
\hline \multirow{2}{*}{ Alta } & Sim & $91,4 \pm 30,9$ & $105,4 \pm 39,3^{\text {cd }}$ & $121,0 \pm 43,6^{\mathrm{ac}}$ & $10,5 \pm 13,3^{\mathrm{ab}}$ \\
\hline & Não & $75,3 \pm 25,3$ & $79,1 \pm 32,1^{\mathrm{bc}}$ & $112,1 \pm 31,3^{c}$ & $5,5 \pm 5,3^{a}$ \\
\hline \multirow{3}{*}{6 meses } & Sim & $81,0 \pm 8,9$ & $137,0 \pm 74,7^{\text {de }}$ & $140,2 \pm 41,1^{\mathrm{a}}$ & $4,5 \pm 3,3^{a}$ \\
\hline & Não & $90,4 \pm 15,6$ & $161,0 \pm 81,5^{e}$ & $135,5 \pm 31,6^{a}$ & $5,2 \pm 4,3^{a}$ \\
\hline & TS & Glicose & Triglicerídeos & Colesterol Total & Insulina \\
\hline \multirow{2}{*}{ Admissão } & Sim & $78,6 \pm 52,7$ & $35,4 \pm 14,6^{a}$ & $84,0 \pm 27,5^{b}$ & $10,4 \pm 16,8^{\mathrm{ab}}$ \\
\hline & Não & $91,2 \pm 43,2$ & $63,0 \pm 37,8^{\mathrm{ab}}$ & $67,4 \pm 14,5^{b}$ & $12,3 \pm 11,4^{b}$ \\
\hline \multirow{2}{*}{ Alta } & Sim & $74,5 \pm 25,2$ & $76,4 \pm 29,2^{\mathrm{bc}}$ & $115,0 \pm 32,8^{a}$ & $5,3 \pm 5,4^{\mathrm{ab}}$ \\
\hline & Não & $90,5 \pm 29,9$ & $106,2 \pm 40,2^{\text {cd }}$ & $114,6 \pm 40,3^{a}$ & $10,1 \pm 12,3^{\mathrm{ab}}$ \\
\hline \multirow{2}{*}{6 meses } & Sim & $90,2 \pm 15,9$ & $158,8 \pm 75,6^{e}$ & $139,6 \pm 36,1^{c}$ & $4,8 \pm 4,2^{\mathrm{a}}$ \\
\hline & Não & $82,8 \pm 10,5$ & $144,6 \pm 87,6^{\text {de }}$ & $132,3 \pm 31,5^{\mathrm{ac}}$ & $5,3 \pm 3,8^{\mathrm{ab}}$ \\
\hline
\end{tabular}

Legenda:

- $\quad \mathrm{O}^{2}$ - uso de oxigênio na alta da UTIN

- $\quad$ TS - transfusão sanguínea.

- $\quad$ * Quando as médias foram estatisticamente diferentes nos três períodos avaliados mediante o teste de ANOVA são representadas na tabela pela junção das letras ab; ac; bc; cd; de;

- $\quad$ ** Quando as médias não foram estatisticamente significativas nos três períodos avaliados são representadas na tabela pelas letras a; b; c; d ou e isoladamente.

- Valores de referência utilizados: Glicemia $80-126 \mathrm{mg} / \mathrm{dL}$ (pós-prandial); Triglicerídeos <85mg/dL (sem jejum); Colesterol Total <170mg/dL (sem jejum); Insulina 2,6 a 24,9 uUI/mL.

Os tratamentos de transfusão sanguínea e uso de oxigênio não influenciaram nos perfis lipídico, glicêmico e insulínico dos RNPT ( $p=>0,05)$. Analisando isoladamente os valores de glicose, observou-se que apresentaram estabilidade em todos os períodos estudados, independente do tratamento a que o RNPT foi submetido. Os valores da insulina decresceram a partir do momento de admissão e se mostraram estáveis da alta até o sexto mês (Tabela 5).

Os níveis de triglicerídeos e colesterol tendem a aumentar ao longo do tempo nos dois grupos de pacientes avaliados, ou seja, entre os que realizaram e os que não realizaram os tratamentos, bem como na presença ou não de comorbidades (Tabela 4 e 5).

\section{DISCUSSÃO}

Em relação à caracterização dos RNPT estudados, observou-se que dados semelhantes foram encontrados em outros estudos nacionais ${ }^{(10-11)}$. O perfil glicêmico identificado nos RNPT é referido na literatura como esperado, ou seja, concentrações neonatais de glicose diminuem após o nascimento, uma vez que logo após o nascimento, a resposta dos hormônios que regulam os níveis de glicose no sangue é menos sensível devido à imaturidade do monofosfato de adenosina cíclico, um segundo mensageiro relacionado ao metabolismo da glicose. Além disso, RNPT tem menor capacidade de armazenamento de glicogênio do que recém-nascidos a termo. Portanto, a hipoglicemia pós-natal é comum entre os RNPT(12).

Estudos acerca do perfil lipídico, glicêmico e de insulina de RNPT não são comuns na literatura, não se tendo valores padronizados para esse grupo. Em recém-nascidos saudáveis é comum encontrar taxas glicêmicas baixas com valores de $30 \mathrm{mg} / \mathrm{dl}$ por até uma a duas horas após o nascimento. Referente à definição de hiperglicemia não 
há consenso na literatura, sendo frequentemente definida como glicemia $>125 \mathrm{mg} / \mathrm{dL}$ (6,9 mmol/L) ou glicose plasmática $>150 \mathrm{mg} / \mathrm{dL}$ ( $8,3 \mathrm{mmol} / \mathrm{L})$. No entanto, esses níveis são frequentemente observados durante infusões de glicose em recém-nascidos, especialmente naqueles extremamente prematuros e podem não indicar morbidade, o que não exige intervenção terapêutica ${ }^{(13)}$. Como a secreção de insulina é estimulada pela glicose no mecanismo de maturação, a concentração média de glicose em recém-nascidos sem complicações de saúde ocorre em torno das 72 horas de vida, quando passa a ser semelhante àquelas crianças mais velhas ${ }^{(14)}$.

Em relação à insulina, os valores de referências em indivíduos adultos seriam de 2,6 a $24,9 \mathrm{Ul} / \mathrm{ml}^{(15)}$, conforme padronização do método de análise do laboratório em que foi realizado o exame de insulina. Hiperinsulinemia é considerada quando os valores estão acima de $15 \mathrm{mU} / \mathrm{mL}$ em crianças $^{(16)}$. A primeira defesa do organismo do neonato é a supressão da secreção de insulina quando a concentração de glicose plasmática cai abaixo da média pós-absortiva normal de $85 \mathrm{mg} / \mathrm{dL}^{(14)}$.

Por essa razão os valores de concentração da insulina nos RNPT começaram a cair ao longo do seguimento, para manter a concentração plasmática de glicose estável. Considera-se, nessa pesquisa, que a maior concentração de insulina na admissão na UTIN pode ser advinda do uso de glicocorticoide ante parto utilizado comumente para estimular a maturidade pulmonar do RNPT ao nascer. Estudo ${ }^{(17)}$ identificou que os níveis de insulina foram maiores em bebês pequenos para idade gestacional quando comparados aos adequados a idade gestacional.

Na presente pesquisa, o perfil lipídico dos RNPT demonstrou elevação gradual da admissão aos seis meses de IC. Em estudo ${ }^{(18)}$ realizado com RNPT de 28 a 37 semanas e RN a termo, utilizando amostra de sangue de cordão, os pesquisadores encontraram aumento da taxa de colesterol Low Density Lipoproteins (LDL) em RNPT, comparando aos a termo. Outra investigação(19) de seguimento observou que RNPT tinham triglicerídeos aumentados no primeiro ano de vida, comparados aos a termo.

A prematuridade tem sido associada ao desenvolvimento de alguns comprometimentos de saúde como intolerância à glicose, dislipidemias e hipertensão arterial, tanto em crianças como em adultos ${ }^{(2)}$. Corroborando essa afirmação, pesquisa(20) indica que elevadas taxas de triglicerídeo em RNPT aumentam o risco do desenvolvimento de doenças cardiovasculares futuras. Ainda, estudo desenvolvido na índia encontrou que os perfis lipídicos em recém-nascidos apresentam índices mais elevados do que em recém-nascidos de outras partes do mundo, demonstrando taxa de colesterol total de 103,92 $\pm 47,79$ e triglicérides de 187,62 $\pm 144,44 \mathrm{mg} / \mathrm{dl}^{(21)}$, dados próximos aos de nosso estudo.

Ao analisar a média isolada dos níveis de triglicerídeos de RNPT com sepse tardia, DBP e para os RNPT sem presença de ROP, observa-se média maior que os valores de referência indicados ${ }^{(9)}$, em que para a faixa etária entre zero e nove anos é considerado valor elevado o colesterol $>170 \mathrm{mg} / \mathrm{dl}$ sem jejum, > $75 \mathrm{mg} / \mathrm{dl}$ com jejum e $>85 \mathrm{mg} / \mathrm{dl} \mathrm{sem} \mathrm{jejum} \mathrm{para} \mathrm{triglicerídeos.} \mathrm{Por} \mathrm{não} \mathrm{haver} \mathrm{valores} \mathrm{de} \mathrm{referência} \mathrm{para} \mathrm{RNPT} \mathrm{teve-se} \mathrm{que} \mathrm{utilizar} \mathrm{os}$ valores de referência de crianças e adolescentes de dois a 19 anos e nessa análise, os RNPT aos seis meses de idade corrigida apresentaram resultados de triglicerídeos e colesterol mais elevados que a referência utilizada. Assim, podem ser compreendidos como grupo de risco para o desenvolvimento de Síndrome Metabólica (SM) e doenças cardiovasculares, visto que os valores de triglicerídeos e colesterol apresentam-se elevados aos seis meses de $I C^{(4,19)}$. 
RNPT, especialmente os de muito baixo peso e prematuros extremos recebem como um dos tratamentos na UTIN, a nutrição parenteral, o que contribui para a trigliceridemia ${ }^{(22)}$. Estudo aponta que doses mais elevadas de aminoácidos na hospitalização podem levar a distúrbios metabólicos precoces, os quais apresentam valores mais elevados em lactentes nascidos extremamente prematuros ${ }^{(23)}$. Contudo, pesquisas que abordem o perfil lipídico de prematuros são recentes, o que indica a necessidade do desenvolvimento de mais estudos nessa área.

A insulina apresentou comportamento distinto nos casos de sepses tardia e ECN, ambas as morbidades oriundas de processos infeciosos. Nesses casos, a homeostase encontra-se ameaçada por microrganismos invasores. Como reação, o organismo busca estabelecer resposta complexa em que diversos mediadores neuroendócrinos e inflamatórios estão envolvidos, sendo a hiperglicemia uma característica importante das alterações agudas que ocorrem durante essa resposta, além de ser uma resposta à resistência à insulina, que impede a glicose de fazer parte do ciclo de Krebs. Esse processo implica em modificações no metabolismo de carboidratos, dentre eles tem-se a resistência periférica à insulina, glicogenólise hepática aumentada e gliconeogênese aumentada, visando redirecionar o fornecimento de energia aos órgãos vitais(24).

Com base no processo fisiológico de resposta à infecções pode-se explicar o comportamento distinto da concentração de insulina quando feita a comparação entre RNPT com e sem ECN ou sepse, em que se observou maior concentração nos RNPT portadores desses problemas. Para tanto, durante o período de internação na UTIN a equipe pode incluir a dosagem de insulina como rotina para os RNPT que desenvolverem ECN e sepse tardia, para conduzir tratamentos que reduzam esse aumento. Em relação à sepse precoce não houve correlação positiva com alteração do perfil insulínico.

Consideram-se como limitações deste estudo: o tamanho da amostra e o curto período de seguimento, indicando a necessidade de acompanhamento dos RNPT ao longo dos primeiros anos de vida, incluindo as avaliações de perfil metabólico para atestar os achados aqui identificados.

\section{CONCLUSÕES}

Ao correlacionar as variáveis comorbidades e tratamentos recebidos com o perfil bioquímico ao longo do seguimento entre os RNPT que tinham alguma comorbidade e que foram submetidos a transfusão sanguínea e uso de oxigênio na alta da UTIN com aqueles que não estiveram sob essas mesmas condições, identificou-se que os níveis de triglicerídeos e colesterol tendem a aumentar ao longo do tempo. A concentração plasmática da glicose apresentou tendência de estabilidade nos RNPT em todos os períodos estudados, realizando ou não os tratamentos.

A ECN e sepse tardia foram as comorbidades que apresentaram diferença estatística significativa nos níveis de insulina, o que pode sugerir que a elevação da insulina pode-se constituir em marcador precoce de sepse. A partir desse conhecimento, pode-se inserir o controle de insulina como rotina na UTIN, além de rigoroso controle glicêmico em RNPT pela enfermagem, antes destes desenvolverem essas comorbidades, principalmente, em caso de sepse, uma vez que a hiperglicemia é decorrente da resistência à insulina, indicando a necessidade de intervenção.

A partir desse estudo, foi possível classificar os RNPT como de potencial risco para o desenvolvimento de complicações metabólicas e doenças cardiovasculares em fases posteriores da vida, devido às alterações lipídicas e insulínicas encontradas. Desse modo, devem ser realizados esforços de acompanhar tais neonatos desde o parto 
até o seguimento, reconhecendo precocemente alterações metabólicas e propondo intervenções que reduzam o risco do desenvolvimento de complicações na idade adulta.

Compreende-se que os resultados desse estudo contribuem para alertar a equipe de saúde para a necessidade de controles glicêmicos, avaliação de perfil lipídico e insulínico e uso de aminoácidos em alta concentração no manejo do RNPT durante a hospitalização, bem como no seguimento após a alta hospitalar, quando se deve evitar sobrecarga glicêmica e lipídica que venham a contribuir para alterações em longo prazo. Mediante as evidências deste estudo, faz-se urgente desenvolver mais pesquisas com a população de RNPT para se obter valores de referência para esse grupo, uma vez que na literatura os valores são para crianças maiores e adolescentes.

\section{REFERÊNCIAS}

1. Leal MD, Esteves-Pereira AP, Nakamura-Pereira M, Torres JA, Theme-Filha M, Domingues RM, et al. Prevalence and risk factors related to preterm birth in Brazil. Reprod Health [Internet]. 2016 [acesso em: 05 dez. 2018];13(Suppl 3):127. Disponível em: https://doi.org/10.1186/s12978-016-0230-0.

2. Marciniak A, Patro-Małysza J, Kimber-Trojnar Ż, Marciniak B, Oleszczuk J, Leszczyńska-Gorzelak B. Fetal programming of the metabolic syndrome. Taiwan J Obstet Gynecol [Internet]. 2017 [acesso em: 05 dez. 2018];56(2):133-8. Disponível em: https://doi.org/10.1016/j.tjog.2017.01.001.

3. Ribeiro AM, Lima MC, Lira PIC, Silva GAP. Baixo peso ao nascer e obesidade: associação causal ou casual? Rev Paul Pediatr [Internet]. 2015 [acesso em: 05 dez. 2018];33(3):340-8. Disponível em: https://doi.org/10.1016/j.rpped.2014.09.007.

4. Huang YT, Lin HY, Wang CH, Su BH, Lin CC. Association of preterm birth and small for gestational age with metabolic outcomes in children and adolescents: A population-based cohort study from Taiwan. Pediatr Neonatol [Internet]. 2018 [acesso em: 05 dez. 2018];59(2):147-153. Disponível em: https://doi.org/10.1016/i.pedneo.2017.07.007.

5. Oliveira MA, Luiza VL, Tavares NU, Mengue SS, Arrais PS, Farias MR, et al. Access to medicines for chronic diseases in Brazil: a multidimensional approach. Rev Saude Publica. 2016 [acesso em: 05 dez. 2018];50(suppl 2):6s. Disponível em:

https://doi.org/10.1590/S1518-8787.2016050006161.

6. Executive Summary. New Dir Youth Dev [Internet]. 2013 [acesso em: 05 dez. 2018];2013(137):11-4. Disponível em: https://doi.org/10.1002/yd.20044.

7. Fenton TR, Kim JH. A systematic review and meta-analysis to revise the Fenton growth chart for preterm infants. BMC Pediatr [Internet]. 2013 [acesso em: 05 dez. 2018];13:59. Disponível em: https://doi.org/10.1186/1471-2431-13-59.

8. Sociedade Brasileira de Diabetes. Diretrizes da Sociedade Brasileira de Diabetes (2015-2016) [Internet]. Rio de Janeiro: A.C. Farmacêutica, 2016 [acesso em: 05 dez. 2018]. Disponível em: https://www.diabetes.org.br/profissionais/images/docs/DIRETRIZESSBD-2015-2016.pdf.

9. Sociedade Brasileira de Análises Clinícas. Consenso Brasileiro para a Normatização da Determinação Laboratorial do Perfil Lipídico [Internet]. 10 dez. 2016 [acesso em: 05 dez. 2018]. Disponível em: http://www.sbac.org.br/acompanhamentopolitico/consenso-brasileiro-para-a-normatizacao-da-determinacao-laboratorial-do-perfil-lipidico/.

10. Rover MMS, Viera CS, Silveira RC, Guimarães ATB, Grassiolli S. Risk factors associated with growth failure in the follow-up of very low birth weight newborns. J Pediatr (Rio J) [Internet]. 2016 [acesso em: 05 dez. 2018];92(3):307-13. Disponível em: https://doi.org/10.1016/j.jped.2015.09.006.

11. Rover MDMS, Viera CS, Toso BRGO, Grassiolli S, Bugs BM. Growth of very low birth weight preterm until 12 months of corrected age. J Hum Growth Dev [Internet]. 2015 [acesso em: 05 dez. 2018];25(3):351-6. Disponível em:

https://doi.org/10.7322/jhgd.90228.

12. Yoon JY, Chung HR, Choi CW, Yang SW, Kim BII, Shin CH. Blood glucose levels within 7 days after birth in preterm infants according to gestational age. Ann Pediatr Endocrinol Metab [Internet]. 2015 [acesso em: 05 dez. 2018];20(4):213-9. Disponível em: https://doi.org/10.6065/apem.2015.20.4.213.

13. Szymońska I, Jagła M, Starzec K, Hrnciar K, Kwinta P. The incidence of hyperglycaemia in very low birth weight preterm newborns. Results of a continuous glucose monitoring study--preliminary report. Dev Period Med [Internet]. 2015 [acesso em: 05 dez. 2018];XIX(3 Pt 1):305-12. Disponível em: http://medwiekurozwoj.pl/articles/2015-3-1-9.pdf.

14. Thornton PS, Stanley CA, De Leon DD, Harris D, Haymond MW, Hussain K, et al. Recommendations from the Pediatric Endocrine Society for Evaluation and Management of Persistent Hypoglycemia in Neonates, Infants, and Children. J Pediatr [Internet]. 2015 [acesso em: 05 dez. 2018];167(2):238-45. Disponível em: https://doi.org/10.1016/j.jpeds.2015.03.057.

15. Biovel. Laboratório Biovel Análises e Pesquisas Clínicas - Home [Internet]. [acesso em: 05 dez. 2018]. Disponível em:

https://www.biovel.com.br/\#exames. 
16. Kostovski M, Simeonovski V, Mironska K, Tasic V, Gucev Z, Metabolic profiles in obese children and adolescents with insulin resistance. Open Access Maced J Med Sci [Internet]. 2018 [acesso em: 05 dez. 2018];6(3):511-8. Disponível em:

https://doi.org/10.3889/oamjms.2018.097.

17. Payal V, Jora R, Sharma P, Gupta PK, Gupta M. Premature birth and insulin resistance in infancy: A prospective cohort study. Indian J Endocrinol Metab [Internet]. 2016 [acesso em: 05 dez. 2018];20(4):497-505. Disponível em: https://doi.org/10.4103/22308210.183470.

18. Li L, Hua J, Jian-Ping H, Yan L. Association between the Lipid Levels and Single Nucleotide Polymorphisms of ABCA1, APOE and HMGCR Genes in Subjects with Spontaneous Preterm Delivery. PLoS One [Internet]. 2015 [acesso em: 05 dez.

2018];10(8):e0135785. Disponível em:: https://doi.org/10.1371/journal.pone.0135785.

19. de Jong M, Cranendonk A, van Weissenbruch MM. Components of the metabolic syndrome in early childhood in very-low-birthweight infants and term small and appropriate for gestational age infants. Pediatr Res [Internet]. 2015 [acesso em: 05 dez.

2018];78(4):457-61. Disponível em: https://doi.org/10.1038/pr.2015.118.

20. Tank S, Jain SK. Altered cord blood lipid profile, insulin resistance \& growth restriction during the perinatal period \& its potential role in the risk of developing cardiovascular disease later in life. Indian J Med Res [Internet]. 2016 [acesso em: 05 dez.

2018];144(2):151-3. Disponível em: https://doi.org/10.4103/0971-5916.195021.

21. Ramaraj SM, Bharath AP, Sanjay KM. Lipid profile in neonates and its relation with birth weight and gestational age. Indian J Pediatr [Internet]. 2015 [acesso em: 05 dez. 2018];82(4):375-7. Disponível em: https://doi.org/10.1007/s12098-014-1661-7. 22. Raman M, Almutairdi A, Mulesa L, Alberda C, Beattie C, Gramlich L. Parenteral Nutrition and Lipids. Nutrients [Internet]. 2017 [acesso em: 05 dez. 2018];9(4):388. Disponível em: https://doi.org/10.3390/nu9040388.

23. Lee BS. Nutritional strategy of early amino acid administration in very low birth weight infants. Korean J Pediatr [Internet]. 2015 [acesso em: 05 dez. 2018];58(3):77-83. Disponível em: https://doi.org/10.3345/kjp.2015.58.3.77.

24. Hellström A, Ley D, Hansen-Pupp I, Hallberg B, Löfqvist C, van Marter L, et al. Insulin-like growth factor 1 has multisystem effects on foetal and preterm infant development. Acta Paediatr [Internet]. 2016 [acesso em: 05 dez. 2018];105(6):576-86.

Disponível em: https://doi.org/10.1111/apa.13350. 